\title{
The Evaluation of Vision Related Quality of Life in Patients with Retinitis Pigmentosa after Suprachoroidal Umbilical Cord Derived Mesenchymal Stem Cell Treatment
}

\author{
Neslihan Sinim Kahraman, Ayse Oner \\ Department of Ophthalmology, Kayseri Acibadem Hospital, Kayseri, Turkey \\ Email: neslihansinim@gmail.com, ayseozoner@gmail.com
}

How to cite this paper: Kahraman, N.S. and Oner, A. (2021) The Evaluation of Vision Related Quality of Life in Patients with Retinitis Pigmentosa after Suprachoroidal Umbilical Cord Derived Mesenchymal Stem Cell Treatment. Open Journal of Ophthalmology, 11, 203-213.

https://doi.org/10.4236/ojoph.2021.113016

Received: June 3, 2021

Accepted: July 30, 2021

Published: August 3, 2021

Copyright $\odot 2021$ by author(s) and Scientific Research Publishing Inc. This work is licensed under the Creative Commons Attribution International License (CC BY 4.0).

http://creativecommons.org/licenses/by/4.0/

\begin{abstract}
Background: The aim of this study was to evaluate vision related quality of life (VRQoL) in patients with retinitis pigmentosa (RP) after suprachoroidal umbilical cord derived mesenchymal stem cell (UC-MSC) treatment. Methods: The patients were evaluated regarding to the VRQoL before the treatment and at the end of the first year. The study was performed in an affiliated hospital of a university between 2018 and 2020. The patients were operated by a single surgeon and evaluated at baseline and at first, sixth and twelfth month after stem cell implantation. To assess patients' subjective visual situation, we used Impact of Vision Impairment (IVI) Profile 28-item questionnaire. It basically evaluated vision-related activities in three subscales: "reading and accessing information", "mobility and independence" and "emotional well-being". This test is established to assess VRQoL in low vision patients. Results: A total of 123 people, ranging in age from 18 to 48 years, participated in the study and $43.9 \%(\mathrm{n}=54)$ were women. The study patients were followed up for one year and the questionnaire was filled by patients at baseline and one year after surgery. In all groups, no difficulty was observed in understanding the questionnaire. The results showed significant improvements in VRQoL after stem cell treatment $(\mathrm{p}<0.05)$. The analysis of each subscale score including "reading and accessing information", "mobility and independence" and "emotional well-being" before and after treatment showed significant improvements in all subscale scores $(\mathrm{p}<0.05)$. Conclusions: IVI 28 item questionnaire seems to be an effective test for the assessment of VRQoL in low vision patients. The suprachoroidal implantation of UC-MSC for the treatment of RP can improve the quality of life of these patients.
\end{abstract}




\section{Keywords}

Stem Cell Therapy, Retinitis Pigmentosa, Umbilical Cord Derived Mesenchymal Stem Cells, Vision Related Quality of Life

\section{Introduction}

Retinitis pigmentosa (RP) is a heterogeneous and progressive group of inherited degenerative diseases which cause loss of photoreceptor cells of the retina. The disease primarily affects rod photoreceptors and deteriorates night vision and peripheral visual field (VF). With the involvement of cone photoreceptors in the advanced stage, central vision decreases. The deterioration of VF can vary between $2.9 \%$ to $8.1 \%$ per year. Finally total blindness can occur between fourth and fifth decades of life in most of the patients [1].

Patients with RP often have difficulties with daily activities. Most of them have difficulties in navigation, orientation, and obstacle detection. Among different measures of visual function, VF area has been shown to be the best predictor of poor mobility in patients with RP. However, measuring visual acuity and VF may incompletely demonstrate the patient's subjective experiences of the daily life. To evaluate the subjective impact of RP to patients' life, visual function questionnaires (VFQs) would be useful [2] [3] [4].

So far there is no curative treatment to prevent the progression of the disease and improve visual function. Current management of the disease aims to slow down the progression by nutritional support, to treat complications, to provide rehabilitation and psychosocial support. New therapeutic options like retinal implants, gene and stem cell therapy are under investigation to decrease progression rate and restore vision [5] [6] [7] [8].

Stem cells are known to have neuroprotective effects by secreting various types of trophic factors. The most common used stem cell type is umbilical cord-derived mesenchymal stem cell (UC-MSC) which has paracrine and immunomodulatory effects by producing trophic factors. Previous studies showed that UC-MSCs are effective in preventing retinal degeneration and increasing photoreceptors survival [9] [10] [11].

There are various ways of stem cell delivery to the eye in clinical studies including subretinal, intravitreal, suprachoroidal, subtenon and intravenous application routes. The results of the largest clinical trial showed the safety and efficacy of suprachoroidal implantation of UC-MSCs in patients with RP [12]. In this current study, our aim was to evaluate the effect of stem cell surgery to vision-related quality of life (VRQoL) by using 28-item Impact of Vision Impairment (IVI) Profile test which was previously validated in our population [13].

\section{Methods}

Ethical Approval: The study was performed in accordance with the Declaration 
of Helsinki. Ethics Committee of the University (2017/480, 10.13.2017) and Review Board of Stem Cell Applications of the Ministry of Health (Registration number: 56733164/203) were completed according to the regulations in our country. All participants of the study were informed and written informed consent was obtained.

Study Design and Setting: This is the second report of a prospective open label phase III clinical study. The first one which contains the six-month results of suprachoroidal UC-MSCs implantation in patients with RP was published previously [12].

The study was performed in an affiliated hospital of a university between 2018 and 2020. The patients were operated by a single surgeon. The patients were evaluated regarding to the VRQoL before the treatment and at the end of the first year. Sample size was set at minimum 70 subjects according to the statistical calculations. After receiving a complete medical history, the patients were evaluated for eligibility according to the inclusion and exclusion criteria. Inclusion criteria were: 1) Snellen visual acuity of $6 / 12$ or worse in the eye with best corrected visual acuity (BCVA) 2) A clinical diagnosis of RP confirmed by optical coherence tomography (OCT) and VF test 3) Age older than 18 years. The exclusion criteria for all the participants were cognitive and mental dysfunction. The technical details of surgery and the preparation of stem cells were published in the previous study of our group [12]. The patients were evaluated at baseline and at first, sixth and twelfth month after stem cell implantation.

Variables and Outcomes: Detailed ophthalmic examination including BCVA and intraocular pressure measurements, anterior segment evaluation, color fundus photography, OCT and VF were performed. Objective assessment could be carried out by these tests but it is also important to assess patients' experience. For this reason, VRQoL of patients by IVI questionnaire was recorded before and one year after stem cell therapy.

\section{VRQoL questionnaire:}

To assess patients' subjective visual status, we used IVI questionnaire. This test has been developed and validated at Centre for Eye Research Australia (CERA). The test is established to assess VRQoL in low vision patients [13] [14] [15] [16].

The IVI questionnaire consists of 28 items with three specific subscales: reading and accessing information, mobility and independence, and emotional well-being (Table 1). The response options for each item are based on a scale of 3 (not at all), 2 (a little), 1 (moderately) to 0 (a lot), with additional response choice of not applicable (do not do this activity for other reasons) for the item 1-15. It is recommended to use Rasch analysis by CERA so in this study we studied with the Rasch-scaled 28-item version of the IVI [14].

In a recent study, the validation of this test was performed after Turkish IVI modification and it was found that the test could detect subjects with low vision and differentiate them from healthy persons. The study reported that the Turkish 
Table 1. Demographic characteristics of all patients included in the study.

\begin{tabular}{|c|c|}
\hline Parameters & \\
\hline \multicolumn{2}{|l|}{ Age (Years) } \\
\hline Mean \pm SD & $36.3 \pm 11.22$ \\
\hline Range & 18 to 48 \\
\hline \multicolumn{2}{|l|}{ Gender, $\mathrm{n}(\%)$} \\
\hline Male & $69(56.1 \%)$ \\
\hline Female & $54(43.6 \%)$ \\
\hline \multicolumn{2}{|l|}{ Job status (\%) } \\
\hline Employed & $73(59 \%)$ \\
\hline Unemployed & $31(25 \%)$ \\
\hline Retired & $19(16 \%)$ \\
\hline \multicolumn{2}{|l|}{ Education } \\
\hline Elementary school & $13(10 \%)$ \\
\hline High school degree & $75(61 \%)$ \\
\hline University degree & $35(29 \%)$ \\
\hline \multicolumn{2}{|l|}{ Age of disease onset (Years) } \\
\hline Mean \pm SD & $19.4 \pm 8.9$ \\
\hline \multicolumn{2}{|l|}{ Family history, n (\%) } \\
\hline Positive & $73(59 \%)$ \\
\hline Negative & $30(25 \%)$ \\
\hline Unknown & $20(16 \%)$ \\
\hline \multicolumn{2}{|l|}{ Inheritance pattern, $\mathrm{n}(\%)$} \\
\hline Autosomal dominant & $29(24 \%)$ \\
\hline Autosomal recessive & $87(71 \%)$ \\
\hline $\mathrm{X}$ linked & $7(5 \%)$ \\
\hline
\end{tabular}

version of the IVI questionnaire is a reliable and valid tool to determine VRQoL in subjects with low vision of various retinal diseases [17].

\section{Statistical analysis}

For the validity of the questionnaire, the Rasch analysis was performed as suggested by the developers of the questionnaire. Differences between groups were evaluated using analysis of variance (ANOVA) and paired t-test. A p value less than 0.05 was significant. The correlation between items was measured by Pearson's correlation coefficient and the internal consistency was analyzed by the Cronbach's alpha coefficient.

\section{Results}

A total of 123 people, ranging in age from 18 to 48 years (mean \pm SD, $36.3 \pm$ 11.22 years), participated to the study and $43.9 \%(n=54)$ were women. The 
mean age at onset of disease was 19.8 years. There was a family history in $59 \%$ of the patients. Among the patients with a family history autosomal dominant pattern was found in $24 \%$, autosomal recessive pattern was found in $71 \%$ and $\mathrm{X}$ linked pattern was found in $5 \%$ of the patients. Demographic data of the patients was shown in Table 1.

When we evaluate BCVA of the study patients, we found a statistically significant improvement after treatment $(\mathrm{p}<0.05)$. Intraocular pressure measurements did not differ during the study period ( $\mathrm{p}>0.05$ ). Anterior segment evaluation showed phthisis bulbi in two (1\%) eyes due to previous complicated surgeries, 68 (28\%) eyes were pseudophacic, 76 (31\%) eyes had varying degrees of posterior subcapsular cataract and $98(40 \%)$ eyes were normal. None of the eyes received cataract surgery during the study period. OCT evaluations showed abnormalities in $15(6 \%)$ patients before surgery. $13(5 \%)$ had cystoid macular edema (CME), 1 (0.4\%) had epiretinal membrane and $1(0.4 \%)$ had vitreomacular traction (VMT). VMT in one patient and CME in 7 patients resolved after surgery. There were no morphological changes in OCT scans of other patients. The mean central macular thickness (CMT) measurements of all treated eyes did not differ after treatment $(\mathrm{p}>0.05)(118.6 \pm 52.7 \mu \mathrm{m}$ before treatment and 123.0 $\pm 50.6 \mu \mathrm{m}$ after treatment).

The study patients were followed up for one year and questionnaire was filled by patients at baseline and one year after surgery. A member of our team read and filled the questionnaire for the participants with inadequate visual acuity for reading. In all groups, no difficulty was observed in understanding the questionnaire. The results showed significant improvements in VRQoL after stem cell treatment. The details of the results can be seen in Table 2 and Table $3(p<0.05)$.

The mean scores of each IVI item before and after treatment are all listed in Table 2. Lower values indicate lower visual ability and suggest that the subject is more disabled. When we evaluate each item individually 10 items showed significant improvements after stem cell therapy. The three of the improved items were "looking after appearance", "safety at home" "spilling or breaking things". The remaining 7 items were related to the "emotional wellbeing" of the patient. The analysis on each subscale score including "reading and accessing information", "mobility and independence" and "emotional well-being" before and after treatment showed significant improvements in all subscale scores. (Table 3) ( $\mathrm{p}<$ 0.05). Regarding to the IVI test subscale scores, the lowest score was obtained in "reading and accessing information" subgroup. The highest score was in "mobility" and "independence" questions. There were statistically significant improvements in BCVA and VF mean deviation (VF-MD) results after treatment $(\mathrm{p}<0.05)$. There were correlations between BCVA, VF-MD values and subscale scores before and after treatment.

\section{Discussion}

Patients with RP usually express concerns about limitations in vision-related 
daily activities including reading, driving and independent mobility. They also have emotional concerns related to vision loss and its' effect on their social function. When determining the impact of low vision on individuals, it is important

Table 2. Mean scores of all items of IVI questionnaire in all subjects.

\begin{tabular}{|c|c|c|c|c|}
\hline & $\begin{array}{c}\text { Before Treatment } \\
\text { Mean } \pm \text { SD }\end{array}$ & $\begin{array}{l}\text { After Treatment } \\
\text { Mean } \pm \text { SD }\end{array}$ & $\begin{array}{c}\text { Control } \\
\text { Mean } \pm \text { SD }\end{array}$ & $P$ value \\
\hline 1: (R) Looking after appearance & $.91 \pm 1.03$ & $1.28 \pm 1.04$ & $3.0 \pm 0.0$ & $\mathrm{P}<0.05^{\star *}$ \\
\hline 2: (R) Opening package & $1.31 \pm 1.08$ & $1.59 \pm 1.01$ & $3.0 \pm 0.0$ & $\mathrm{P}>0.05^{*}$ \\
\hline 3: (R) Getting information & $1.60 \pm 1.05$ & $1.68 \pm 1.00$ & $3.0 \pm 0.0$ & $\mathrm{P}>0.05^{*}$ \\
\hline 4: (R) Handling money & $1.44 \pm 1.13$ & $1.60 \pm 1.05$ & $3.0 \pm 0.0$ & $\mathrm{P}>0.05^{*}$ \\
\hline 5: (M) Recreational activities & $1.40 \pm 1.04$ & $1.60 \pm 0.94$ & $3.0 \pm 0.0$ & $\mathrm{P}>0.05^{*}$ \\
\hline 6: (M) Getting outdoors in familiar environments & $1.47 \pm 1.10$ & $1.63 \pm 1.10$ & $3.0 \pm 0.0$ & $\mathrm{P}>0.05^{*}$ \\
\hline 7: (M) Getting outdoors in unfamiliar environments & $1.81 \pm 1.18$ & $1.88 \pm 1.22$ & $3.0 \pm 0.0$ & $\mathrm{P}>0.05^{*}$ \\
\hline 8: (M) Travelling or using transport & $1.45 \pm 1.13$ & $1.51 \pm 1.08$ & $3.0 \pm 0.0$ & $\mathrm{P}>0.05^{*}$ \\
\hline 9: (M) Walking on uneven ground & $1.64 \pm 1.20$ & $1.68 \pm 1.16$ & $3.0 \pm 0.0$ & $\mathrm{P}>0.05^{*}$ \\
\hline 10: (M) Crossing the street & $1.43 \pm 1.23$ & $1.58 \pm 1.15$ & $3.0 \pm 0.0$ & $\mathrm{P}>0.05^{*}$ \\
\hline 11: (M) Safety at home & $1.08 \pm 1.16$ & $1.44 \pm 1.12$ & $3.0 \pm 0.0$ & $\mathrm{P}<0.05^{\star *}$ \\
\hline 12: (M) Spilling or breaking things & $1.13 \pm 0.91$ & $1.44 \pm 0.89$ & $3.0 \pm 0.0$ & $\mathrm{P}<0.05^{* *}$ \\
\hline 13: (M) Burning or scalding yourself & $1.52 \pm 1.11$ & $1.71 \pm 1.02$ & $3.0 \pm 0.0$ & $\mathrm{P}>0.05^{*}$ \\
\hline 14: (M) Having a fall & $1.16 \pm 0.90$ & $1.37 \pm 0.91$ & $3.0 \pm 0.0$ & $\mathrm{P}>0.05^{*}$ \\
\hline 15: (M) Safety outside the home & $1.35 \pm 1.12$ & $1.40 \pm 1.10$ & $3.0 \pm 0.0$ & $\mathrm{P}>0.05^{*}$ \\
\hline 16: (M) Going down steps, stairs, or curb & $1.22 \pm 1.04$ & $1.45 \pm 1.02$ & $3.0 \pm 0.0$ & $\mathrm{P}>0.05^{*}$ \\
\hline 17: (E) Felt embarrassed & $1.56 \pm 1.29$ & $1.72 \pm 1.18$ & $3.0 \pm 0.0$ & $\mathrm{P}>0.05^{*}$ \\
\hline 18: (E) Felt frustrated or annoyed & $1.00 \pm 1.08$ & $1.22 \pm 1.12$ & $3.0 \pm 0.0$ & $\mathrm{P}>0.05^{*}$ \\
\hline 19: (E) Felt lonely or isolated & $1.64 \pm 1.26$ & $1.78 \pm 1.08$ & $3.0 \pm 0.0$ & $\mathrm{P}>0.05^{*}$ \\
\hline 20: (E) Felt sad or low & $0.85 \pm 1.08$ & $1.18 \pm 1.08$ & $3.0 \pm 0.0$ & $\mathrm{P}<0.05^{* *}$ \\
\hline 21: (E) Worried about eyesight & $0.95 \pm 1.06$ & $1.17 \pm 1.05$ & $3.0 \pm 0.0$ & $\mathrm{P}>0.05^{\star}$ \\
\hline 22: (E) Interference with the relationships with family & $2.02 \pm 1.26$ & $2.13 \pm 1.16$ & $3.0 \pm 0.0$ & $\mathrm{P}>0.05^{*}$ \\
\hline 23: (E) Felt like a nuisance or a burden & $1.24 \pm 1.22$ & $1.59 \pm 1.19$ & $3.0 \pm 0.0$ & $\mathrm{P}<0.05^{* *}$ \\
\hline 24: (E) Felt vulnerable & $1.12 \pm 1.20$ & $1.47 \pm 1.14$ & $3.0 \pm 0.0$ & $\mathrm{P}<0.05^{\star *}$ \\
\hline 25: (E) Stopped doing something & $1.20 \pm 0.96$ & $1.48 \pm 0.93$ & $3.0 \pm 0.0$ & $\mathrm{P}<0.05^{\star *}$ \\
\hline 26: (E) Needed help from other people & $1.10 \pm 0.95$ & $1.35 \pm 1.01$ & $3.0 \pm 0.0$ & $\mathrm{P}<0.05^{* *}$ \\
\hline 27: (E) Treated in the wrong way & $1.54 \pm 1.02$ & $1.78 \pm 0.99$ & $3.0 \pm 0.0$ & $\mathrm{P}<0.05^{\star *}$ \\
\hline 28: (E) Interfered with life in general & $1.18 \pm 1.09$ & $1.45 \pm 1.06$ & $3.0 \pm 0.0$ & $\mathrm{P}<0.05^{\star *}$ \\
\hline Total Score & $18.66 \pm 20.84$ & $22.00 \pm 23.91$ & $84 \pm 0.0$ & $\mathrm{P}<0.05^{* *}$ \\
\hline
\end{tabular}

IVI, Impact of Vision Impairment; R, Reading and accessing information; M, Mobility and independence; E, Emotional well-being. Data are expressed as mean $\pm \mathrm{SD}$. ${ }^{*}$ Although the mean scores of the items showed no significant difference after treatment, the scores of all items before and after treatment were significantly lower than the controls. ${ }^{*}$ The mean scores of these items were significantly higher than the baseline. However, the scores of all items before and after treatment were significantly lower than the controls. 
Table 3. Visual acuity, visual field mean deviation, subscale scores of controls and patients before and after treatment.

\begin{tabular}{ccccc}
\hline & Before Treatment & After Treatment & Control & p value \\
\hline Visual Acuity (Snellen Lines) & $0.22 \pm 0.17$ & $0.36 \pm 0.11$ & $0.96 \pm 0.06$ & $<0.05^{*}$ \\
VF-MD Value (dB) & $28.18 \pm 3.18$ & $26.11 \pm 4.26$ & $2.28 \pm 2.32$ & $<0.05^{\star}$ \\
IOP (mmHg) & $14.10 \pm 2.12$ & $15.2 \pm 2.68$ & $16.12 \pm 2.89$ & $>0.05$ \\
Reading and accessing information & $3.774 \pm 3.93$ & $7.87 \pm 8.78$ & 30.0 & $<0.05^{*}$ \\
Mobility and independence & $10.04 \pm 9.70$ & $11.24 \pm 9.77$ & 18.0 & $<0.05^{\star}$ \\
Emotional well-being & $9.39 \pm 10.44$ & $11.24 \pm 10.46$ & 36.0 & $<0.05^{*}$ \\
\hline
\end{tabular}

Data were expressed as mean \pm SD. VF-MD: Visual field mean deviation. dB: Decibel. ${ }^{\star}$ The scores of all subscale groups were significantly lower than the controls. There was a statistically significant improvement in all subscale groups after stem cell treatment.

to quantify its impact on these individuals' daily life, activities and emotional status [18]. Although 25-item National Eye Institute Visual Function Questionnaire (NEI VFQ-25) is the most widely used assessment tool for VRQoL, IVI-28 item seems to be a better instrument for low vision populations. The findings of this instrument provide an opportunity for a more detailed measurement of the effects of different types of low-vision rehabilitation programs and therapies [13] [14].

The results of a cross-validation study in our population showed that the Turkish-version of the IVI questionnaire is a reliable and valid tool to measure VRQoL in patients with low vision of various underlying conditions. The subscales of the questionnaire also revealed high internal consistencies and high test-retest reliability in the test-retest subgroup of the patients and controls [17].

Previous studies showed that the reading performance is impaired in most patients with RP and it correlates with contrast sensitivity, visual acuity, and VF [15] [16]. The first four questions of the IVI questionnaire were related to the ability of reading and getting information. The mean scores of these four individual items were significantly lower than the controls. The mean subscale score was also significantly lower than the controls. Our results confirmed that VA and VF-MD values showed a correlation with the mean subscale scores related to the reading ability. It is reported that highly demanding visual tasks, such as reading, are impaired when visual acuity is lower than 20/30 (0.60 decimal) [15]. The mean VA in our study was lower than the mentioned value above $(0.22$ Snellen lines) therefore the impairment in the reading ability was an expected result.

It is known that RP patients have difficulties in orientation, finding products, performing indoor and outdoor activities and using public transport. Mobility is reported to be impaired at visual acuity of 20/200 [15] [16] [19]. IVI questionnaire included 12 items which gave detailed information about mobility and independence ability of the patients. Although the mean VA in our study was better than the mentioned value above, the mean scores of the questions were sig- 
nificantly lower than the controls. Patients may have difficulties in mobility even in better visual acuity levels. It is thought that the constriction in the visual field with a mean VF-MD value of $28.18 \mathrm{~dB}$, contributed to the impairment in mobility abilities of our patients.

The third part of the IVI questionnaire is related to the emotional status. RP is emotionally devastating to the patients due to their gradual vision loss. RP patients are more vulnerable to depression, some studies have addressed the issues of depression and the VRQoL in this population [19] [20]. Depressive symptoms are also associated with poor mental wellbeing and lower levels of general happiness with life [21]. The IVI questionnaire includes 12 questions about emotional status of the patients. The scores of these 12 items were significantly lower than the controls. These results confirmed that patients with RP felt embarrassed, frustrated, annoyed, sad, lonely and vulnerable. They were worried about their eyesight. They had problems about the relationships with the family members. They needed help from other people and they were treated in the wrong way because of their decreased visual acuity.

Stem cell-based therapies are one of the most recent therapeutic options for irreversible degenerative diseases like RP. MSCs are known to provide trophic support for neuroprotection and regeneration of damaged retinal cells [11] [12]. In a recent report, 124 eyes of $82 \mathrm{RP}$ patients received UC-MSCs implantation to the suprachoroidal area and had no serious systemic or ocular complications. This study also showed statistically significant improvements in visual acuity, VF and mfERG tests during the 6-month follow-up period [12].

To evaluate the therapeutic efficacy of treatment options, as well as the clinical assessment with VA and ophthalmological tests, it is important to measure the effect of the treatment on VRQoL. There is only one study in the literature evaluating the effect of stem cell treatment on VRQoL. In this clinical trial, which is called Reticell, the investigators assessed the VRQoL in patients with RP submitted to intravitreal use of bone marrow-derived stem cells with NEI VFQ-25 [22]. Twenty patients were scheduled to answer the questionnaire before treatment and 3 and 12 months after treatment. The outcomes of the study showed that the cell therapy with intravitreal use of bone marrow-derived stem cells could improve the quality of life of patients with $\mathrm{RP}$, although the improvement was lost with time. In this study the study group was small, the researchers reported total score of the questionnaire and there was no detailed information about the items individually.

Our study showed that stem cell treatment induced improvement in all three parts of the questionnaire including "reading and accessing information", "mobility and independence" and "emotional well-being". When we evaluate the items individually although the scores of all items were higher than the baseline after treatment, only 10 of them were statistically significant. Seven of the 10 items were related to the emotional status and 2 of them were related to the mobility and independence of the patients. It is known that there is a significant 
psychological component in low vision patients. Patients have high expectations with respect to the recent developing treatments. We believe that the idea and availability of a new therapeutic option may have a positive influence on psychological and emotional status of the patients. Previous studies reported a correlation between quality of life and the sensitivity of the retina in patients with RP [22] [23]. In a study including 108 RP patients, Seo et al. [24] measured BCVA, VF and collected the self-reported NEI-VFQ 25. They calculated functional vision score (FVS) by using the functional field score (FFS) and the functional acuity score (FAS). The results showed that FVS was highly correlated to the BCVA, FFS and FAS. They emphasize that, the remaining functional field is important for VRQoL especially in advanced RP patients. We believe that the improvement in BCVA and VF may also enhance VRQoL and emotional wellbeing of the patients in our study.

In conclusion, IVI 28 item test seems to be effective for the assessment of VRQoL in low vision patients. The suprachoroidal implantation of UC-MSC for the treatment of RP can improve the quality of life of these patients.

\section{Limitations}

There were several limitations to this study.

1) This study included patients with medium and late phase of RP and mean BCVA was 0.22 Snellen lines. The stem cell therapy and the IVI-28 item test would be more beneficial in the early stage of the disease.

2) Patients may have distinct environmental, cultural, or geographic characteristics. Therefore VR-QOL measured with IVI-28 item test may not be generalizable to other RP patients.

3) Some of the abilities mentioned in the test may have been affected due to other health problems or co-occurring eye conditions.

4) Findings may also have been influenced by the interviewer.

\section{Acknowledgements}

We thank the participants of the study. We would like to thank Prof. Dr. Ercument Ovali and the staff members of Acibadem Labcell for providing the stem cells. We also thank the staff members of Acibadem Kayseri Hospital for their contribution to the study tests.

\section{Funding}

This research did not receive any specific grant from funding agencies in the public, commercial or not-for-profit sectors.

\section{Authors' Contributions}

NS: Patient follow-up, data collection, manuscript preparation.

AO: Study design, patient selection and follow-up, surgical intervention, data collection, manuscript preparation. 
All authors read and approved the manuscript.

\section{Conflicts of Interest}

The authors declare no conflicts of interest regarding the publication of this paper.

\section{References}

[1] Grover, S., Fishman, G.A., Anderson, R., Alexander, K.R. and Derlacki, D.J. (1997) Rate of Visual Field Loss in Retinitis Pigmentosa. Ophthalmology, 104, 460-465. https://doi.org/10.1016/S0161-6420(97)30291-7

[2] Black, A., Lovie-Kitchin, J.E., Woods, R.L., Arnold, N., Byrnes, J. and Murrish, J. (1997) Mobility Performance in Retinitis Pigmentosa. Clinical and Experimental Optometry, 80, 1-12. https://doi.org/10.1111/j.1444-0938.1997.tb04841.x

[3] Haymes, S., Guest, D., Heyes, A. and Johnston, A. (1996) Mobility of People with Retinitis Pigmentosa as a Function of Vision and Psychological Variables. Optometry and Vision Science, 73, 621-637. https://doi.org/10.1097/00006324-199610000-00001

[4] Costela, F.M., Pesudovs, K., Sandberg, M.A., Weigel-DiFranco, C. and Woods, R.L. (2020) Validation of a Vision-Related Activity Scale for Patients with Retinitis Pigmentosa. Health and Quality of Life Outcomes, 18, 196.

https://doi.org/10.1186/s12955-020-01427-8

[5] Yaylacioglu Tuncay, F., Guntekin Ergun, S., Oner, A., Turan, A., Ozmert, E., Ergun, M.A. and Ozdek, S. (2020) Inherited Eye Diseases in Turkey: Current Approaches and Future Directions. Seminars in Medical Genetics, Part $C$ of the American Journal of Medical Genetics, 184, 773-781. https://doi.org/10.1002/ajmg.c.31829

[6] Oner, A. (2017) Recent Advancements in Gene Therapy for Hereditary Retinal Dystrophies. Turkish Journal of Ophthalmology, 47, 338-343.

https://doi.org/10.4274/tjo.41017

[7] Oner, A. (2018) Stem Cell Treatment in Retinal Diseases: Recent Developments. Turkish Journal of Ophthalmology, 48, 33-38. https://doi.org/10.4274/tjo.89972

[8] Limoli, P.G., Vingolo, E.M., Limoli, C. and Nebbioso, M. (2019) Stem Cell Surgery and Growth Factors in Retinitis Pigmentosa Patients: Pilot Study after Literature Review. Biomedicines, 7, E94. https://doi.org/10.3390/biomedicines7040094

[9] Zhang, W., Wang, Y.X., Kong, J.H., Dong, M., Duan, H.T. and Chen, S. (2017) Therapeutic Efficacy of Neural Stem Cells Originating from Umbilical Cord-Derived Mesenchymal Stem Cells in Diabetic Retinopathy. Scientific Reports, 7, Article No. 408. https://doi.org/10.1038/s41598-017-00298-2

[10] Huo, D.M., Dong, F.T., Yu, W.H., Gao, F. (2010) Differentiation of Mesenchymal Stem Cell in the Microenviroment of Retinitis Pigmentosa. International Journal of Ophthalmology, 3, 216-219.

[11] Özmert, E. and Arslan, U. (2020) Management of Retinitis Pigmentosa by Wharton's Jelly Derived Mesenchymal Stem Cells: Preliminary Clinical Results. Stem Cell Research \& Therapy, 11, 25. https://doi.org/10.1186/s13287-020-1549-6

[12] Kahraman, N.S. and Oner, A. (2020) Umbilical Cord Derived Mesenchymal Stem Cell Implantation in Retinitis Pigmentosa: A 6-Month Follow-Up Results of a Phase 3 Trial. International Journal of Ophthalmology, 13, 1423-1429.

https://doi.org/10.18240/ijo.2020.09.14 
[13] Lamoureux, E.L., Pallant, J.F., Pesudovs, K., Rees, G., Hassell, J.B. and Keeffe, J.E. (2007) The Impact of Vision Impairment Questionnaire: An Assessment of Its Domain Structure Using Confirmatory Factor Analysis and Rasch Analysis. Investigative Ophthalmology \& Visual Science, 48, 1001-1006.

https://doi.org/10.1167/iovs.06-0361

[14] Lamoureux, E.L., Pallant, J.F., Pesudovs, K., et al. (2006) The Impact of Vision Impairment Questionnaire: An Evaluation of İts Measurement Properties Using Rasch Analysis. Investigative Ophthalmology \& Visual Science, 47, 4732-4741.

https://doi.org/10.1167/iovs.06-0220

[15] Virgili, G., Pierrottet, C., Parmeggiani, F., Pennino, M., Giacomelli, G., Steindler, P., Menchini, U. and Orzalesi, N. (2004) MNREAD Charts. Reading Performance in Patients with Retinitis Pigmentosa: A Study Using the MNREAD Charts. Investigative Ophthalmology \& Visual Science, 45, 3418-3424.

https://doi.org/10.1167/iovs.04-0390

[16] Herse, P. (2005) Retinitis Pigmentosa: Visual Function and Multidisciplinary Management. Clinical and Experimental Optometry, 88, 335-350. https://doi.org/10.1111/j.1444-0938.2005.tb06717.x

[17] Kahraman, N.S., Sevim, D.G. and Oner, A. (2019) Cross-Validation of the Turkish Version of the 28-Item Impact of Vision Impairment Profile Test. Open Journal of Ophthalmology, 9, 194-202. https://doi.org/10.4236/ojoph.2019.94021

[18] Prem Senthil, M., Khadka, J. and Pesudovs, K. (2017) Seeing through Their Eyes: Lived Experiences of People with Retinitis Pigmentosa. Eye, 31, 741-748. https://doi.org/10.1038/eye.2016.315

[19] Latham, K., Baranian, M., Timmis, M.A., Fisher, A. and Pardhan, S. (2017) Relative Difficulties of Daily Living Tasks with Retinitis Pigmentosa. Optometry and Vision Science, 94, 317-328. https://doi.org/10.1097/OPX.0000000000001046

[20] Sainohira, M., Yamashita, T., Terasaki, H., Sonoda, S., Miyata, K., Murakami, Y., Ikeda, Y., Morimoto, T., Endo, T., Fujikado, T., Kamo, J. and Sakamoto, T. (2018) Quantitative Analyses of Factors Related to Anxiety and Depression in Patients with Retinitis Pigmentosa. PLoS ONE, 13, e0195983. https://doi.org/10.1371/journal.pone.0195983

[21] Moschos, M., Chatzirallis, A. and Chatziralli, I. (2015) Psychological Aspects and Depression in Patients with Retinitis Pigmentosa. European Journal of Ophthalmology, 25, 459-462. https://doi.org/10.5301/ejo.5000590

[22] Siqueira, R.C., Messias, A., Messias, K., et al. (2015) Quality of Life in Patients with Retinitis Pigmentosa Submitted to Intravitreal Use of Bone Marrow-Derived Stem Cells (Reticell-Clinical Trial). Stem Cell Research \& Therapy, 6, 29. https://doi.org/10.1186/s13287-015-0020-6

[23] Arcieri, R.S., Messias, K., Castro, V.M., Siqueira, R.C., Jorge, R. and Messias, A. (2013) Intravitreal Autologous Bone-Marrow Stem Cells in Retinitis Pigmentosa Patients: One-Year Results. ARVO 2013. Investigative Ophthalmology \& Visual Science, 54, E-Abstract 643.

[24] Seo, J.H., Yu, H.G. and Lee, B.J. (2009) Assessment of Functional Vision Score and Vision-Specific Quality of Life in İndividuals with Retinitis Pigmentosa. Korean Journal of Ophthalmology, 23, 164-168. https://doi.org/10.3341/kjo.2009.23.3.164 